\title{
Composición química de semillas de chía, linaza y rosa mosqueta y su aporte en ácidos grasos omega-3
}

\section{Chemical composition of chia seed, flaxseed and rosehip and its contribution in fatty acids omega-3}

Paula Jiménez $P$. (1)

Lilia Masson S. (2)

\begin{abstract}
The aim of this study was to characterize oilseeds: chia (Salvia hispanica), flax (Linum usitatissimum) and rosehip (Rosa rubiginosa), according to its chemical composition and oil analysis. Fatty acid composition, content of tocols and oxidative stability was determined in the oil from these seeds. The chemical composition was characterized by a high content of fat in chia and flaxseed, high content of protein in chia and flaxseed, and high content of dietary fiber in rosehip. The fatty acid composition consisted mostly of polyunsaturated: linolenic in chia and flaxseed, linoleic in rosehip. Tocols content and oxidative stability were higher in rosehip compared to the other oils. The results show that the oils studied could be used as potential sources of functional ingredients like omega-3 fatty acids.

Key words: oils, omega-3 fatty acids, chia, flaxseed, rosehip.
\end{abstract}

\section{INTRODUCCIÓN}

Los componentes de reserva de las semillas consisten en proteínas, carbohidratos y lípidos. La proporción relativa y localización de estos compuestos varía de acuerdo a la especie (1). Las semillas, en general, son fuente de compuestos lipídicos que incluyen ácidos grasos, tocoferoles, triglicéridos, fosfolípidos, esfingolípidos y esteroles (2).

Los lípidos son nutrientes importantes en la dieta tanto humana como animal, entre sus componentes, destacan los ácidos grasos poliinsaturados de cadena larga (AGPICL), los cuales son componentes dietarios que participan en múltiples procesos fisiológicos, cumpliendo un rol estructural en los fosfolípidos de las membranas celulares y son sustratos para la síntesis de diversos mediadores que modelan múltiples procesos como inmunidad, patologías infecciosas y enfermedades inflamatorias.

Entre los AGPICL encontramos dos grupos principales; los ácidos grasos omega-3 (w-3) y omega-6 (w-6), los cuales son ácidos grasos esenciales para el ser humano debido a que carecemos de la maquinaria enzimática necesaria para biosintetizarlos y por lo tanto son aportados por la dieta. El primer exponente de los ácidos grasos omega-3 es el ácido a-linolénico (C18:3), el cual vía desaturasas y elongasas se
Vilma Quitral R. (1)

(1) Departamento de Nutrición. Facultad de Medicina. Universidad de Chile, Santiago, Chile. (2) Departamento de Ciencia de los Alimentos y Tecnología Química. Facultad de Ciencias Químicas y Farmacéutica. Universidad de Chile, Santiago, Chile.

$$
\begin{array}{r}
\text { Dirigir la correspondencia a: } \\
\text { Dra. Paula Jiménez P. } \\
\text { Departamento de Nutrición } \\
\text { Facultad de Medicina. Universidad de Chile } \\
\text { Av. Independencia 1027 } \\
\text { Santiago, Chile. } \\
\text { Fono: 9786758 } \\
\text { E-mail: paulajimenez@med.uchile.cl }
\end{array}
$$

Este trabajo fue recibido el 26 de Marzo de 2013 y aceptado para ser publicado el 7 de Mayo de 2013.

puede transformar en el ácido eicosapentaenoico (C20:5, EPA) y posteriormente en el ácido docosahexaenoico (C22:6, DHA). A su vez, el primer exponente de los ácidos grasos w- 6 es el ácido linoleico (C18:2) y uno de sus derivados más importantes es el ácido araquidónico (C20:4, AA) (3, 4). El AA, el EPA y el DHA son importantes componentes estructurales de los fosfolípidos de las membranas y son el sustrato para la formación de una serie de derivados lipídicos Ilamados eicosanoides. Los ácidos EPA y DHA pueden ser aportados por la dieta (preformados), encontrándose en pescados, mariscos y algas o a partir de su precursor de origen vegetal. El ácido linolénico, precursor de DHA, tiene baja disponibilidad a partir de la dieta, siendo muy restringido su consumo en algunas poblaciones. Actualmente los cambios en los patrones de alimentación han provocado un mayor consumo de aceites vegetales ricos en ácido linoleico $(w-6)$, por lo que la relación w-6/w-3 es del orden de 15:1 en muchos países, cuando la relación ideal es 5:1 o máximo 10:1 (5).

La utilización de semillas con aceites de alto contenido de ácido linolénico o del aceite mismo en alimentación es una herramienta interesante para aumentar el aporte de ácidos grasos $\mathrm{w}$-3 a la dieta. Una alternativa la constituyen los aceites de semillas de linaza (Linum usitatissimum), rosa mosqueta (Rosa 
rubiginosa) y chía (Salvia hispanica), los cuales se caracterizan por un alto contenido de ácido linolénico en su composición.

De acuerdo a estos antecedentes, el objetivo de este estudio fue caracterizar semillas de oleaginosas (rosa mosqueta, linaza y chía), desde el punto vista químico proximal y analizar el aceite de estas materias primas, como ingredientes con ácidos grasos omega 3.

\section{MATERIAL Y MÉTODOS}

Las semillas de chía (Salvia hispánica), linaza (Linum usitatissimum) y rosa mosqueta (Rosa rubiginosa) fueron adquiridas en un supermercado de la Región Metropolitana, Santiago, Chile. Se trasladaron al laboratorio, donde fueron analizadas inmediatamente.

\section{Análisis químicos}

Para la determinación de la composición química de las semillas, las muestras fueron analizadas en cuanto al contenido de humedad, proteína total, cenizas y fibra dietética (6). El contenido de hidratos de carbono disponibles se determinó por diferencia. El contenido de grasa en las semillas se extrajo por Soxhlet (7).

La composición de ácidos grasos se determinó por cromatografía gas líquido (GLC), usando un cromatógrafo Hewlett Packard 5890 serie II, analizándolos como ésteres metílicos derivatizados (8).

Se determinaron los tocoferoles en las muestras de aceite provenientes de las semillas por HPLC con detector de fluorescencia, de acuerdo al método Ce 8-89 (7).

La estabilidad de los aceites se evaluó determinando el tiempo de inducción, de acuerdo al método oficial Cd 12b92 (7). Para estos ensayos se utilizó un equipo Rancimat 679 Metrohm AG. Herisau (Suiza), a $110{ }^{\circ} \mathrm{C}$, con un flujo de aire constante de $20 \mathrm{~mL} / \mathrm{min}$.

Los resultados se analizaron con análisis de varianza (ANOVA) y prueba de rango múltiple de Duncan con un nivel de significancia de un 95\%. Se utilizó el Programa SPSS 15.0

\section{RESULTADOS Y DISCUSIÓN}

En la tabla 1 se presenta la composición química de semillas, expresados en $\mathrm{g} / 100 \mathrm{~g}$. Existen diferencias significativas $(p<0.05)$ entre las semillas para los componentes analizados.

Las semillas vegetales analizadas se caracterizan por su bajo contenido de humedad. En el caso de semillas de chía y linaza, además de tener un alto contenido de materia grasa, también contienen un aporte proteico importante. A diferencia de rosa mosqueta, que a pesar de aportar menor cantidad de materia grasa y proteína en relación a las otras semillas en estudio, presentó un alto contenido de fibra dietética (64 $\mathrm{g} / 100 \mathrm{~g})$. Con respecto a la composición química de semilla de linaza determinada en el presente estudio, ésta es similar a las que describieron Mueller et al. (9) en variedades café y amarilla, en cuanto al contenido de humedad (7.4 y 7.3 g/100g, respectivamente) y proteínas (21.7 y $21.6 \mathrm{~g} / 100 \mathrm{~g}$, respectivamente), pero menor en cuanto a materia grasa (41.9 y $40.8 \mathrm{~g} / 100 \mathrm{~g}$, respectivamente) y mayor para el contenido de hidratos de carbono totales. Por otra parte, bases de datos de USDA reportan valores de humedad, proteínas y fibra dietética similares a los determinados en el presente estudio (10).

En el caso de la semilla de chía, Weber et al., (11) y más tarde Ayerza y Coates, (12) determinaron un contenido de proteínas que varió entre 19 a 27 g/100g y 16 a 26 g/100g, (respectivamente), rangos en los que se encuentra el contenido de proteínas determinados en el presente estudio. De acuerdo a estos resultados, cabe destacar que esta semilla contiene un mayor contenido de proteínas en relación a otras semillas de consumo habitual como es el caso de trigo, maíz, arroz, avena, cebada y amaranto (13). Respecto al contenido de materia grasa, los valores reportados por otros autores fluctúan entre 30.0 y 33.5 g/100g $(12,14)$, mientras que las muestras analizadas en este estudio contienen un valor levemente inferior de $27.9 \mathrm{~g} / 100 \mathrm{~g}$.

Con respecto a la semilla de rosa mosqueta y a diferencia de chía y linaza, en la literatura se encuentran muy pocos estudios que han determinado la composición química de esta semilla, así Turgut et al., (15) y Camilo, (16) reportaron contenidos de proteína, lípidos, cenizas, fibra e hidratos de carbono totales similares a los determinados en este estudio.

Las diferencias encontradas en la composición química de las tres semillas se pueden atribuir a distintos factores, entre los cuales se puede mencionar a la especie, variedad, lugar geográfico, estacionalidad y otros factores agronómicos (17).

La tabla 2 presenta la composición de los principales ácidos grasos de los aceites de semillas de chía, linaza y rosa de mosqueta.

Los aceites de las tres semillas estudiadas se caracterizaron por un alto contenido de ácidos grasos poliinsaturados, destacando la presencia de los ácidos grasos esenciales (linoleico y linolénico), con un promedio entre ambos de un $70 \%$ aproximadamente. Los aceites de las tres semillas contienen ácido oleico, siendo mayor en los aceites de rosa mosqueta $(16,12 \%)$ y linaza $(15,76 \%)$ que en chía $(8,9)$. En los aceites de semilla de chía y linaza se identificó principalmente ácido linolénico (51.8 y 55.3\%, respectivamente). El aceite de rosa mosqueta en cambio presenta mayoritariamente ácido linoleico en su composición con $41.6 \%$, y el ácido linolénico

TABLA 1

Composición química de semillas (g/100g)

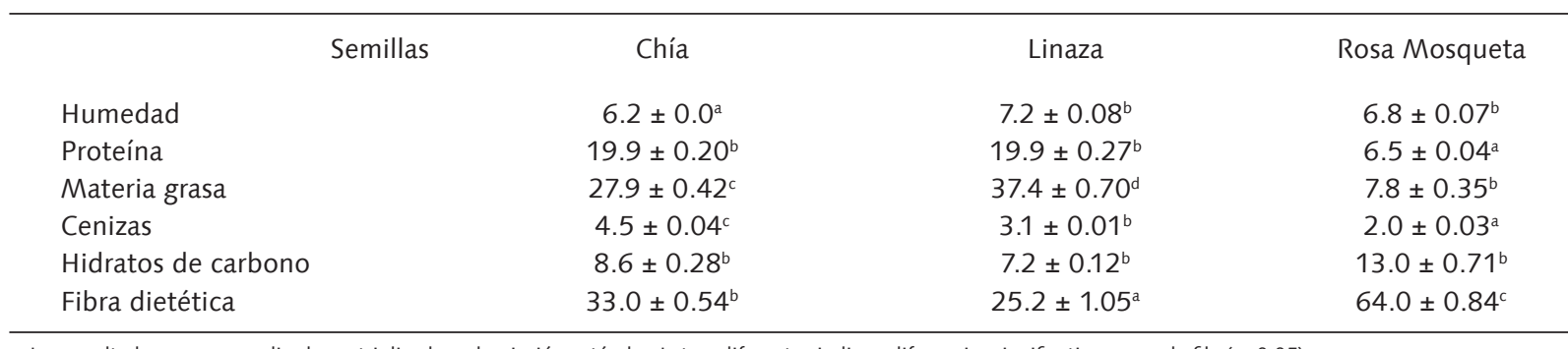

Los resultados son promedio de un triplicado \pm desviación estándar. Letras diferentes indican diferencias significativas en cada fila ( $p<0.05$ ). 
también está presente, aunque en menor proporción que en las semillas de chía y linaza, con un $27 \%$. Con respecto al aceite de chía, los principales ésteres metílicos encontrados en este estudio, concuerdan con los reportados por Chicco et al., (18), Peiretti y Gai (19), Ixtaina et al., (20); Ayerza y Coates (12), y Martínez et al., (14), a excepción del ácido linolénico que fue levemente inferior en relación a lo descrito por estos autores $(64.6,64.1,65.6,60.8$ y 60.5\%, respectivamente). Un comportamiento similar, se observó en la composición de ácidos grasos en aceite de linaza, siendo concordante con lo encontrado por otros autores en esta especie cultivada en distintos lugares geográficos, como es el caso de Europa, Canadá, Argentina e India (21, 22), sin embargo el contenido de ácido linolénico determinado en el presente estudio fue mayor que el descrito por Masson y Mella (23). La composición de ácidos grasos de semilla de rosa mosqueta, fue similar a lo descrito por Szentmihályi et al., (24) y Camilo (16), sin embargo, Masson y Mella (23) encontraron valores superiores en ácido linolénico (34.4\%).

Cabe destacar que en general, los aceites de semillas

TABLA 2

Composición de ácidos grasos de los aceites de semillas

Ácidos Grasos

Ác. Laúrico C12:0

Ác. Mirístico C14:0

Ác. Palmítico C16:0

Ac. Heptadecanoico C17:0

Ác. Esteárico C18:0

Ác. Eicosanoico C20:0

Ác. Docosanoico C22:0

Ác. Tetracosanoico C24:0

Acidos grasos saturados totales

Ác.Palmitoleico C16:1

Ác. Elaídico C18:1w9 trans

Ác. Oleico C18:1w9 cis

Ác. Octadecaenoico C18:1 w7 cis

Ác. Octadecaenoico C18:1 isom

Ác. Octadecaenoico C18:1 isom

Ác. Eicosaenoico C20:1

Ác. Eicosaenoico C20:1w9

Ác. Grasos Monoinsat.Totales

Ác.Hexadecadienoico C16:2

Ác. Octadecadienoico C18:2 trans

Ác. Octadecadienoico C18:2 trans

Ác. Octadecadienoico C18:2 trans

Ác. Octadecadienoico C18:2 trans

Ác .Linoleico C18:2w6

Ác. Octadecadienoico C18:2 isom

Ác. Octadecadienoico C18:2 isom

Ác. Octadecadienoico C18:2 isom

Ác. Octadecadienoico C18:2 isom

Ác. Octadecadienoico C18:2 isom

Ác. Eicosadienoico C20:2

Ác.Y-Linolénico C18:3w6

Ác Octadecatrienoico C18:3 isom

Ác Octadecatrienoico C18:3 isom

Ác. Linolénico C18:3w3 cis

Ác. Eicosatrienoico C20:3w3

Ác. Grasos Poliinsat. Totales

No identificados

Relación sat:mono:polinsaturados

Relación w6:w3
Chía

$-$

$0,08 \pm 0,00$

$7,29 \pm 0,17$

$0,03 \pm 0,04$

$3,84 \pm 0,09$

$0,23 \pm 0,00$

Trazas

Trazas

11,47

$0,06 \pm 0,00$

$1,91 \pm 0,29$

$8,91 \pm 0,30$

$1,31 \pm 0,05$

$0,23 \pm 0,03$

$0,17 \pm 0,02$

$0,12 \pm 0,00$

$0,17 \pm 0,00$

12,88

$0,18 \pm 0,00$

$0,48 \pm 0,04$

$0,89 \pm 0,12$

$0,34 \pm 0,04$

$0,16 \pm 0,03$

$19,36 \pm 0,16$

$0,10 \pm 0,01$

$0,08 \pm 0,01$

-

-

-

$0,28 \pm 0,03$

$0,08 \pm 0,01$

$0,32 \pm 0,30$

$0,09 \pm 0,01$

$51,82 \pm 1,49$

74,18

1,47

1: $1,12: 6,47$

0,37
Linaza

$\%$ ésteres metílicos

$$
\begin{gathered}
- \\
0,05 \pm 0,00 \\
5,21 \pm 0,10 \\
0,07 \pm 0,00 \\
3,40 \pm 0,07 \\
0,47 \pm 0,02 \\
0,13 \pm 0,02 \\
0,10 \pm 0,00 \\
9,43
\end{gathered}
$$

$0,39 \pm 0,07$

$15,76 \pm 0,47$

$0,48 \pm 0,06$

-

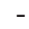

$-$

$0,13 \pm 0,02$ 16,76

$\begin{aligned} 0,20 & \pm 0,04 \\ & - \\ & - \\ & -\end{aligned}$

$16,23 \pm 0,18$

$0,33 \pm 0,10$

$0,16 \pm 0,04$

$0,06 \pm 0,02$

$0,17 \pm 0,02$

$0,04 \pm 0,01$

$0,62 \pm 0,11$

$0,29 \pm 0,08$

$0,11 \pm 0,05$

$55,3 \pm 0,18$

$0,03 \pm 0,00$

73,54

0,27

1: $1,78: 7,8$

0,29
Rosa mosqueta

$0,04 \pm 0,01$

$0,07 \pm 0,01$

$3,71 \pm 0,04$

$2,46 \pm 0,09$

$0,74 \pm 0,01$

$0,18 \pm 0,00$

Trazas

7,20

$0,10 \pm 0,00$

$2,87 \pm 0,87$

$16,12 \pm 0,24$

$0,23 \pm 0,00$

$0,16 \pm 0,02$

$0,12 \pm 0,00$

$0,44 \pm 0,01$

20,04

$0,32 \pm 0,03$

-

-

-

$41,55 \pm 0,44$

$0,86 \pm 0,09$

$0,70 \pm 0,05$

$0,37 \pm 0,04$

-

-

-

$0,49 \pm 0,05$

$0,25 \pm 0,02$

$27,48 \pm 0,49$

72,02

0,74

1: $2,78: 10,0$ 1,51 
vegetales aportan contenidos importantes de ácido linoleico, sin embargo, son pocos las que aportan ácido linolénico, como es el caso de canola, soja, colza y nuez entre otros, con 12, 7, 11 y $10 \mathrm{~g} / 100 \mathrm{~g}$ de aceite respectivamente (25). Por esta razón, es importante conocer otras fuentes de ácido linolénico, no sólo por su esencialidad sino además, por su rol como precursor de EPA y DHA, ya que a ambos ácidos grasos se les han atribuido efectos benéficos en la salud humana debido a que tras su incorporación en la dieta, disminuyen el riesgo de enfermedades cardiovasculares, tienen un efecto positivo en la función cardíaca, la hemodinámica (26), participan activamente en los procesos de formación y funcionamiento neuronal y óptico (27) e inhiben el crecimiento de células cancerígenas y tumorales (28). Sin embargo, un aspecto importante a considerar es que si bien el ácido linolénico es precursor de EPA y DHA, la conversión a estos ácidos grasos es diferente. En animales se ha demostrado que esta conversión es muy rápida a EPA, pero a DHA es menos significativa, aunque hay resultados controversiales en este aspecto (29). Estudios realizados en primates no humanos, indican que la conversión de ácido linolénico a DHA es de $0.23 \%$ a $0.57 \%(30,31)$. Estudios adicionales han demostrado que aproximadamente el $0.2 \%$ del ácido linolénico que ingresa al cerebro sufre conversión a DHA (32). Una investigación realizada en humanos, demostró que al administrar aceite de linaza ( $3 \mathrm{~g}$ ácido linolénico) por 12 semanas aumentó el nivel de EPA (60\%) en plasma, sin producirse cambios en el nivel de DHA (33). En el caso de los recursos marinos, existe evidencia que enzimas desaturasas de peces son similares a la de los mamíferos, describiéndose que éstas tienen preferencia por el sustrato $w-3$, es decir, por ácido linolénico, lo que es muy importante en el contexto de reemplazar parte del aceite de pescado por aceite vegetal en la dieta de peces de cultivo (34).

Los aceites de productos marinos contienen ácidos grasos $\omega-3$ de cadena larga, como EPA y DHA, pero el contenido de aceite es variable. El aceite de alga Ulva lactuca, por ejemplo, contiene $0.87 \%$ de EPA y $2.15 \%$ de DHA, pero como su contenido de aceite es de solo $1.27 \mathrm{~g} / 100 \mathrm{~g}$, lo que realmente aportan $100 \mathrm{~g}$ de alga corresponde a $11.05 \mathrm{mg}$ de EPA y $27.31 \mathrm{mg}$ de DHA. Las algas en general presentan bajo contenido de materia grasa (35). En el caso de peces, las especies grasas presentan altas proporciones de EPA y DHA. El contenido de estos ácidos grasos en el aceite de Jurel (Trachurus murphyi) es de 9.4 y $25.1 \%$ respectivamente. Dentro de los salmónidos cultivados en Chile, el aceite de salmón del Atlántico (Salmo salar) presenta 7.9 y $15.2 \%$ de EPA y DHA, valores similares corresponden al aceite de trucha arcoiris (Oncorhynchus mykiss), con 7.3 y $18.7 \%$ de EPA y DHA (36).

Otro resultado obtenido en este estudio, corresponde a la relación $\omega 6 / \omega 3$ obtenida para los aceites de chía, linaza y rosa mosqueta $(0.37,0.29$ y 1.51 respectivamente). En general, estas fueron menores a 1 , a excepción del aceite de rosa mosqueta, en el cual es esperable esta relación dado su alto contenido de ácido linoleico. Según las recomendaciones nutricionales, la relación ideal es de 5:1 a 10:1 como máximo (5). Sin embargo, esta relación es difícil de obtener directamente de la dieta, ya que esta en general, se caracteriza por un exceso de ácidos grasos omega 6 y un bajísimo aporte de ácidos grasos omega 3, lo que finalmente se refleja en muchas de las patologías que afectan a las personas. Por lo tanto, a través de este estudio, se podría recomendar que para aumentar los aportes de ácidos grasos omega 3, y equilibrar la relación, cualquiera de estos aceites son una alternativa potencial para ser incorporados en la alimentación humana y/o animal.

La tabla 3 presenta el contenido de tocoles en los aceites de semillas de chía, linaza y rosa mosqueta. De los aceites de las tres semillas, el de rosa mosqueta presenta un valor significativamente más alto de tocoferoles, siendo y-tocoferol el isómero más abundante en los aceites de las tres semillas estudiadas, lo que concuerda con lo descrito para otros aceites (37).

En el presente estudio se determinó $423 \mathrm{mg} / \mathrm{kg}$ de tocoferoles en aceite de chía, lo que concuerda con los valores descritos por Ixtaina et al. (20) a partir de semillas de chía provenientes de Argentina y de Guatemala, con valores entre 238 a $427(\mathrm{mg} / \mathrm{kg})$, siendo -tocoferol el principal isómero $(85 \%$ del total, es decir, entre 202 y $363 \mathrm{mg} / \mathrm{kg}$ ) y a-tocoferol entre 0.4 y $9.9 \mathrm{mg} / \mathrm{kg}$, estos contenidos dependieron del sistema de extracción utilizado. Más tarde, estos mismos autores (38), encontraron valores de tocoferoles totales de $480 \mathrm{mg} / \mathrm{kg}$, con $463 \mathrm{mg} / \mathrm{kg}$ de -tocoferol.

El contenido de tocoles en el aceite de linaza fue similar a los que reportan Tuberoso et al. (22), con contenidos totales de $588 \mathrm{mg} / \mathrm{kg}(\alpha=9, \gamma=575$ y $\delta=4)$, pero menores a los encontrados en aceites de linaza prensados en frío (153.5 a 244.7 $\mathrm{mg} / \mathrm{kg}$ de tocoferoles totales y con $\mathrm{\gamma}$-tocoferol entre $106 \mathrm{y}$ $150 \mathrm{mg} / \mathrm{Kg}$ ), estas diferencias en el contenido de tocoferoles podrían atribuirse a la eficiencia del sistema de prensado utilizado. Además, en la muestras de aceite de linaza utilizado en el presente estudio, se identificó otro pico previo y de menor concentración que $y$-tocoferol, que tentativamente y de acuerdo a datos de literatura corresponde a plastocromanol-8 (PC-8) un homólogo derivado de y-tocotrienol, que se caracteriza químicamente por estar compuesto de un anillo 6 cromanol con grupos metilos localizados en la posición 2, 7 y 8 y por una cadena de unidades isoprenoides, esta última más larga en el caso de PC-8 (8 unidades). PC-8 se encuentra sólo en algunos aceites vegetales, tal es el caso de semillas de Brassica napus, Linum sp y Cannabis sativa $(39,40)$ y también en aceites de maíz y soja. A este tocol se le han atribuido importantes propiedades antioxidantes.

TABLA 3

Composición y contenido de tocoles $(\mathrm{mg} / \mathrm{Kg})$, en aceites de semillas

Chía Linaza Rosa mosqueta

a-tocopherol

Y-tocopherol

$\delta$ tocoferol

Plastocromanol- 8 (PC-8)

Total
Linaza

202

976

23

$\begin{array}{cc}323 & 364 \\ & 976 \\ & 23\end{array}$

423

534

1201 
Con respecto al aceite de rosa mosqueta, éste presentó un alto contenido de tocoferoles, especialmente $y$-tocoferol (976 mg/kg), lo que concuerda con datos informados por Camilo (16).

La importancia de los tocoferoles en alimentos y a nivel biológico, se debe a las propiedades antioxidantes de estos compuestos, así se ha visto que sus efectos están relacionados con su carácter protector frente a enfermedades degenerativas crónicas como las enfermedades coronarias (41), degeneración neuronal y aparición de tumores en diferentes localizaciones además de su contribución a la reducción de la peroxidación lipídica (42) y su actividad antihipertensiva.

La tabla 4 presenta los valores de tiempos de inducción determinados por Rancimat a $110^{\circ} \mathrm{C}$, expresados en horas, de los aceites estudiados. El aceite de rosa mosqueta muestra una mayor estabilidad oxidativa, en relación a los aceites de semilla de linaza y chía. Este efecto podría explicarse por su composición en ácidos grasos menos poliinsaturados ya que contiene mayor proporción de ácido graso linoleico (41.6\%) que linolénico (27.5\%), y alto contenido de tocoferoles. La proporción de oxidación en ácidos grasos como esteárico, oleico, linoleico y linolénico, ha sido descrita en 1: 100: 1200: 2500, respectivamente (43). Con respecto al tiempo de inducción del aceite de semilla de chía, éste fue bastante menor (0.8 h) a lo descrito por Ixtaina et al. $(20,38)$ de $2.4 \mathrm{~h}$. Sin embargo, estas diferencias podrían atribuirse a las menores temperaturas utilizadas en los ensayos realizados por estos autores $\left(98^{\circ} \mathrm{C}\right)$.

La estabilidad oxidativa de una matriz lipídica depende de varios factores que incluyen el grado de insaturación de los ácidos grasos, composición de compuestos menores (tocoferoles, carotenoides, entre otros), condiciones medioambientales y presencia de antioxidantes, entre otros (43).

\section{CONCLUSIONES}

En las semillas estudiadas, el contenido de materia grasa presenta el siguiente orden decreciente: linaza > chía > rosa mosqueta. La composición de ácidos grasos que constituyen los aceites de semillas vegetales (chía, linaza y rosa mosqueta), se caracterizaron por estar constituidos principalmente por ácidos grasos altamente poliinsaturados, siendo el ácido linolénico y linoleico, los principales componentes. La incorporación de aceites vegetales altos en ácido linolénico permite mejorar la relación $\omega 6: \omega 3$ y favorecer la conversión a EPA y DHA. Las semillas estudiadas y sus aceites podrían ser utilizados como potenciales fuentes de ingredientes funcionales altos en ácidos grasos omega-3.

\section{RESUMEN}

El objetivo de este estudio fue caracterizar semillas de oleaginosas: chía (Salvia hispanica), linaza (Linum usitatissimum) y rosa mosqueta (Rosa rubiginosa), de acuerdo a la composición química y análisis de sus aceites. Se realizó el análisis químico proximal de las semillas. En los aceites de estas materias primas se determinó la composición en ácidos grasos, contenido de tocoles y estabilidad oxidativa. La composición química se caracterizó por un alto contenido de grasa en chía y linaza, proteína en chía y linaza y fibra dietética en rosa mosqueta. Los ácidos grasos de estos aceites fueron mayoritariamente poliinsaturados: linolénico en chía y linaza, y linoleico en rosa mosqueta. El contenido de tocoles y la estabilidad oxidativa fue mayor en rosa mosqueta. Los resultados muestran que los aceites estudiados podrían ser utilizados como potenciales fuentes de ingredientes funcionales altos en ácidos grasos omega-3.

Palabras clave: aceites, ácidos grasos omega-3, chía, linaza, rosa mosqueta.

\section{BIBLIOGRAFÍA}

1. Baud S, Lepiniec L. Physiological and developmental regulation of seed oil production Review Article. Prog Lipid Res 2010; 49: 235-249.

2. Matthäus $B$, Aitzetmüller $K$, Friedrich $H$. The new database "Seed oil Fatty Acids" (SOFA) Grasas y Aceites 2003; 54: 188-193.

3. Knoch B, Barnett $M$, Roy $N$, McNabb W. Study of the effects of dietary polyunsaturated fatty acids: Molecular mechanisms involved in intestinal inflammation. Grasas Aceites 2009; 60: 8-21.

4. Valenzuela $R$, Tapia G, González M, Valenzuela A. Omega-3 fatty acids (EPA and DHA) and its application in diverse clinical situations. Rev Chil Nutr. 2011; 38: 356-67.

5. FAO/OMS. Fats and Oils in Human Nutrition. Report of a Joint Expert Consultation FAO/OMS. FAO Food and Nutrition 1994. N 57.

6. AOAC. Official Methods of Analysis of the Association of Official Analytical Chemist International; 18th Ed. Maryland, U.S.A. 2005.

7. AOCS. Official methods and recommended practices of the American oil chemists society. 4th Ed. Champaign, IL: AOCS Press. 1993.

8. AENOR. Asociación Española de Normalización. Norma UNE 55037-73, Catálogo de Normas UNE, Madrid. 1991.

9. Mueller $K$, Eisner $P$, Kirchhoff E. Simplified fractionation process for linseed meal by alkaline extraction - Functional properties of protein and fibre fractions. J Food Eng. 2010; 99:49-54.

10. USDA-Database en [http://ndb.nal.usda.gov/ndb/foods/ show/3692?qlookup $=+$ Linum + usitatissimum $\&$ fg $=\&$ forma $t=\& \operatorname{man}=\& /$ facet $=\& \max =25 \&$ new $=1]$

11. Weber C, Gentry H, Kohlhepp E, McCrohan P. The nutritional and chemical evaluation of chia seeds. Ecol Food Nutr. 1991; 26:119-25.

12. Ayerza $R$, Coates W. Protein content, oil content and fatty acid profiles as potential criteria to determine the origin of

\section{TABLA 4}

Tiempo de inducción de aceites de semillas.

Aceite

Chía

Linaza

Rosa mosqueta
Tiempo de Inducción $110^{\circ} \mathrm{C}(\mathrm{h})$

$0.8 \mathrm{a}$

$1.9 \mathrm{~b}$

$3.1 \mathrm{c}$

Letras diferentes indican diferencias significativas $(p<0.05)$. 
commercially grown chia (Salvia hispanica L.). Ind Crop Prod. 2011; 34:1366-71.

13. Capitani M, Spotorno V, Nolasco S, Tomás M. Physicochemical and functional characterization of by-products from chia (Salvia hispanica L.) seeds of Argentina. Food Sci Technol-Leb 2012; 4: 94-102.

14. Martínez M, Marín M, Salgado C, Revol J, Penci M. Chia (Salvia hispanica L.) oil extraction: Study of processing parameters. Food Sci Technol-Leb. 2012; 47:78-82.

15. Turgut L, Havirli A, Kaya A. Effects of various chemical treatments on nutritive value of the rosehip (Rosa canina L.) seed for the ruminant animals. J Animal Vet Adv. 2008; 7:1227-36

16. Camilo C. Análisis proximal de semillas no comunes: Palma Chilena (Jubaea chilensis), Cilantro (Coriandrum sativum), Mora (Rubus glaucus), Rosa mosqueta (Rosa aff. rubiginosa) y caracterización de su aceite. Memoria para optar al título de Ingeniero en Alimentos. Fac. Cs. Qcas y Farmacéuticas, U. de Chile, Chile. 2008. [http://www.tesis.uchile. cl/tesis/uchile/2008/camilo_c/html/index-frames.html]

17. Hidalgo A, Brandolini A, Ratti S. Influence of Genetic and Environmental Factors on Selected Nutritional Traits of Triticum monococcum. J Agric Food Chem 2009; 57: 6342-48.

18. Chicco A, D'Alessandro M, Hein G, Oliva M, Lombardo Y. Dietary chia seed (Salvia hispanica L.) rich in $\alpha$-linolenic acid improves adiposity and normalises hypertriacylglycerolaemia and insulin resistance in dyslipaemic rats. Brit J Nutr. 2008; 101: 41-50.

19. Peiretti P, Gai F. Fatty acid and nutritive quality of chia (Salvia hispanica L.) seeds and plant during growth. Anim Feed Sci Tech. 2009; 148: 267-75.

20. Ixtaina $V$, Martínez M, Spotorno V, Mateo C, Maestri D, Diehl $B$, et al. Characterization of chia seed oils obtained by pressing and solvent extraction. J Food Comp Anal. 2011; 24: 166-74.

21. Van den Berg J, Vermist N, Carlyle L, Holc M, Boon J. Effects of traditional processing methods of linseed oil on the composition of its triacylglycerols. J Sep Sci. 2004; 27: 181-99.

22. Tuberoso I, Kowalczyk A, Sarritzu E, Cabras P. Determination of antioxidant compounds and antioxidant activity in commercial oilseeds for food use. Food Chem. 2007; 103: 1494-501.

23. Masson L, Mella MA. Materias grasas de consumo habitual y potencial en Chile: Composición en ácidos grasos. Facultad de Ciencias Químicas y Farmacéuticas. Universidad de Chile. 1985.

24. Szentmihályi $K$, Vinkler P, Lakatos $B$, Illés $V$, Then $M$. Rose hip (Rosa canina L.) oil obtained from waste hip seeds by different extraction methods. Bioresource Technol 2002; 82:195-201.

25. Jimenez $P$, Masson L, Barriga A, Chávez J, Robert P. Oxidative stability of oils containing olive leaf extracts obtained by pressure, supercritical and solvent-extraction. Eur J Lipid Sci Technol. 2011; 113: 497-505.

26. Kris-Etherton P, Harris W, Appel L. Fish Consumption, Fish Oil, Omega-3 Fatty Acids, and Cardiovascular Disease. Circulation 2002; 106: 2747-57.
27. Innis S, Rioux F, Auestad N, Ackman R. Marine and freshwater fish oil varying in Arachidonic, Eicosapentaenoic and Docosahexaenoic Acids differ in their effects on organ lipids and fatty acids in growing rats. J Nutr. 1995; 125: 2286-93.

28. Hardman W. (n-3) Fatty Acids and Cancer Therapy. J Nutr. 2004;134: 3427S-3430S.

29. Barcelo-Coblijn G, Collison L, Jolly C, Murphy E. Dietary alpha-linolenic acid increases brain but not heart and liver docosahexaenoic acid levels. Lipids 2005; 40: 787-98.

30. Su H, Bernardo L, Mirmiran M, MaXH, Nathanielsz PW, Brenna JT. Dietary $18: 3 n-3$ and $22: 6 n-3$ as sources of 22:6n-3 accretion in neonatal baboon brain and associated organs. Lipids 1999; 34: S347-S50.

31. Su HM, Huang MC, Saad NM, Nathanielsz PW, Brenna JT. Fetal baboons convert $18: 3 n-3$ to $22: 6 n-3$ in vivo. $A$ stable isotope tracer study. J Lipid Res. 2001; 42: 581-6.

32. Demar Jr JC, Ma K, Chang L, Bell JM, Rapoport SI. AlphaLinolenic acid does not contribute appreciably to docosahexaenoic acid within brain phospholipids of adult rats fed a diet enriched in docosahexaenoic acid. J Neurochem. 2005; 94:1063-76.

33. Harper CR, Edwards MJ, DeFilippis AP, Jacobson TA. Flaxseed oil increases the plasma concentrations of cardioprotective (n-3) fatty acids in humans. J Nutr. 2006; 136: 83-7.

34. Vagner $M$, Santigosa E. Characterization and modulation of gene expression and enzymatic activity of delta- 6 desaturase in teleosts: A review. Aquaculture 2011; 315: 131-43.

35. Quitral V, Morales C, Sepúlveda M, Schwartz M. Propiedades nutritivas y saludables de algas marinas y su potencialidad como ingrediente funcional. Rev Chil Nutr. 2012; 39 (4): 196-202.

36. Blanchet $C$, Lucas $M$, Julien P, Morin R, Gingras S, Dewailly E. Fatty Acid Composition of Wild and Farmed Atlantic Salmon (Salmo salar) and Rainbow Trout (Oncorhynchus mykiss). Lipids 2005; 40 (5): 529-31.

37. Szyman'ska R, Kruk J.. Tocopherol content and isomers' composition in selected plant species. Plant Physiol Bioch. 2008; 46:29-33.

38. Ixtaina $V$, Nolasco $S$, Tomás $M$. Oxidative Stability of Chia (Salvia hispanica L.) Seed Oil: Effect of Antioxidants and Storage Conditions. J Am Oil Chem Soc. 2012; 89:1077-90.

39. Kriese U, Schumann E, Weber W, Beyer M, Brühl L, Matthäus $B$. Oil content, tocopherol composition and fatty acid patterns of the seeds of 51 Cannabis sativa L. genotypes. Euphytica 2004; 137: 339-51.

40. Gruszka J, Kruk J. RP-LC for determination of plastochromanol, tocotrienols and tocopherols in plant oils. Chromatographia. 2007; 66: 909-13.

41. Sayago M, Marín I, Aparicio R, Morales M. Vitamina $E$ $y$ aceites vegetales. Grasas Aceites.2007; 58(1):74-86.

42. Wagner $K H$, Tomasch R, Elmadfa I. Impact of diets containing corn oil or olive/sunflower oil mixture on the human plasma and lipoprotein lipid metabolism. Eur J Nutr. 2001; 40: 161-7.

43. Shahidi F, Zhong Y. Lipid oxidation and improving the oxidative stability. Chem Soc Rev. 2010; 39: 4067-79. 